\title{
Long-Term Adherence to a Gluten-Free Diet and Quality of Life of Celiac Patients After Transition to an Adult Referral Center
}

\author{
Annalisa Schiepatti ${ }^{1,2}$ (]) Stiliano Maimaris ${ }^{2} \cdot$ Camila de Queiros Mattoso Archela dos Santos $^{2} \cdot$ Giovanni Rusca $^{2}$. \\ Stefania Costa ${ }^{2} \cdot$ Federico Biagi ${ }^{2}$
}

Received: 31 May 2021 / Accepted: 16 August 2021 / Published online: 15 September 2021

(c) The Author(s) 2021

\begin{abstract}
Background Modalities for the transition to adult care of celiac patients diagnosed during childhood/adolescence and their impact on long-term adherence to a gluten-free diet (GFD-A), quality of life (QOL) and maintenance of follow-up in adulthood are unknown.

Aims To evaluate whether timing of transition affects long-term GFD-A, QOL, and continuity of follow-up in adulthood and to identify predictors of long-term GFD-A.

Methods Clinical and demographic data about pediatric care and adult follow-up at our center were retrospectively collected from clinical notes of celiac patients diagnosed during childhood/adolescence and then referred to our tertiary center. QOL and adult long-term GFD-A were prospectively evaluated with validated questionnaires. These parameters were studied by means of univariate and multivariate statistical analysis.

Results 183 patients $(130 \mathrm{~F}$, mean age at diagnosis $7.6 \pm 5.8$ years) were enrolled. Median age at transition to adult care was 20 years (IQR 17-25). There was no relationship between age at transition to adult care, long-term GFD-A, QOL, and continuity of follow-up. GFD-A tended to improve overall from pediatric care to adult referral (OR 2.92, 95\% CI 1.13-7.87, $p=0.02$ ) and also throughout adult follow-up (OR 9.0, 95\% CI 4.2-19.7, $p<0.01$ ). On multivariable logistic regression analysis, classical symptoms at diagnosis of celiac disease $(p=0.02)$ and good GFD-A at adult referral $(p<0.01)$ predicted good long-term GFD-A, while being lost to follow-up predicted poorer long-term GFD-A $(p=0.02)$.

Conclusions Clinical characteristics can guide development of personalized strategies for implementing long-term GFD-A and ensure maintenance of regular follow-up in celiac patients diagnosed in childhood/adolescence and transitioning to adult care.
\end{abstract}

Keywords Transition $\cdot$ Celiac disease $\cdot$ Gluten-free diet $\cdot$ Follow-up $\cdot$ Quality of life

\section{Introduction}

Celiac disease $(\mathrm{CD})$ is a common chronic immune-mediated enteropathy triggered by dietary gluten in genetically susceptible individuals and characterized by a wide variety of clinical manifestations and an increased mortality compared to the general population [1-5].

Annalisa Schiepatti

annalisa.schiepatti01@universitadipavia.it

1 Department of Internal Medicine and Medical Therapy, University of Pavia, Pavia, Italy

2 Istituti Clinici Scientifici Maugeri, IRCCS, Gastroenterology Unit of Pavia Institute, University of Pavia, Via Salvatore Maugeri 10, 27100 Pavia, Italy
Although in the last decades, $\mathrm{CD}$ has been diagnosed predominantly in adults presenting with non-classical symptoms or even asymptomatically [1-3, 6, 7], pediatric diagnoses still account for a significant proportion of cases $[6,7]$. Although risk of developing pre-malignant/malignant complications is virtually absent in patients diagnosed during childhood/adolescence $[5,8,9]$, delivering a high standard quality of care after transitioning to adulthood and maintenance of long-term adherence to a gluten-free diet (GFD) are crucial for prevention of disease-related morbidity, worsening of quality of life (QOL), and risk of malignant complications [1-3, 10-13]. In this regard, in adults, it has been shown that dietary adherence remains largely stable over follow-up, and when it changes, it usually improves [14]. Pattern of clinical presentation at diagnosis of CD as 
well as cognitive, emotional, and socioeconomic factors has also been shown to influence long-term adherence to a GFD [14-20]. On the contrary, no data are available on long-term dietary patterns and predictors thereof in celiac patients diagnosed in the pediatric setting and then referred to adult care.

Transition from pediatric to adult care is an essential step in the management of chronic diseases. However, for $\mathrm{CD}$, there is a paucity of data regarding the modalities for a successful transition to adult care and how this may influence follow-up, long-term GFD adherence and QOL during adulthood [9, 21-30].

Therefore, the aim of our study is threefold: (1) to provide an overview on the clinical features, long-term GFD adherence, QOL, and continuity of follow-up of patients diagnosed with $\mathrm{CD}$ during childhood/adolescence and then followed-up at an adult tertiary referral center for $\mathrm{CD}$ over a twenty-year period; (2) to evaluate whether timing of transition impacts long-term GFD adherence, QOL, and continuity of follow-up; and (3) to identify predictors of long-term GFD adherence in adulthood.

\section{Patients and Methods}

\section{Study Population and Setting}

This is a single-center longitudinal study spanning 20 years (2000-2020) on celiac patients diagnosed during childhood/ adolescence (age $\leq 18$ years) and then referred to our adult tertiary care center for continuation of follow-up. The study consists of two parts: a retrospective evaluation of clinical and demographic data and a prospective evaluation of longterm GFD adherence, QOL, and continuity of follow-up.

\section{Enrollment Criteria}

In the last 20 years, our center has been providing care to more than 850 adult celiac patients [14]. Of these, only patients who received a diagnosis of $\mathrm{CD}$ in childhood, which we subsequently confirmed upon referral to our institution, were included in the present study. We specify that those few patients directly diagnosed at our center before 18 years old were excluded as by definition they had no transition.

Diagnosis of CD was based on fulfillment of diagnostic criteria in force at the time at which individual patients were diagnosed. In particular, four possible modalities of diagnosis were applied: (a) conventional diagnosis based on villous atrophy (VA) and positive celiac serology [1-3], (b) serological diagnosis in accordance with ESPGHAN guidelines since 2012 [31], (c) diagnostic strategy with duodenal biopsies showing VA at baseline, then histological recovery on a GFD and then recurrence of VA after gluten re-challenge (method of the three diagnostic biopsies) [32], (d) in patients who received a diagnosis of CD in childhood not meeting any of the aforementioned diagnostic criteria, gluten challenge for diagnostic purposes was performed at our center and diagnosis of CD was confirmed on the basis of a certain degree of villous atrophy on duodenal biopsies and positive endomysial antibodies (EmA) [1-3].

\section{Data Collection}

From October to November 2020, clinical and demographic data about pediatric care and adult follow-up at our center were retrospectively collected from patients' clinical notes. These included age at diagnosis of CD, year of diagnosis of $\mathrm{CD}$, presenting symptoms, comorbidities, method of diagnosis [1-3, 31, 32], GFD adherence during pediatric care, age at first referral to our center, results of follow-up duodenal biopsies and celiac-specific antibodies, if performed, GFD adherence at time of transition and during adult follow-up, presence of persistent symptoms despite a GFD, number of medical consultations during adult follow-up, and duration of follow-up at our center.

For the purpose of the prospective part of the study, in December 2020, patients were contacted over the phone to assess current GFD adherence and to offer participation in the QOL study. Anonymous questionnaires to evaluate QOL were subsequently sent by email to patients who consented to participate in the study on QOL. Completed questionnaires were returned by email to the principal investigator.

\section{Criteria to Assess Gluten-Free Diet Adherence and Evaluation of Changes in Gluten-Free Diet Adherence in Adulthood}

GFD adherence was evaluated at different time points. GFD adherence throughout the pediatric period was retrospectively evaluated. This evaluation was based on available medical reports of GFD adherence during pediatric care, as well as by dietary interview with the patient and/or the patient's parents/carers.

After referral, all the patients enrolled in this study had at least two GFD dietary assessments at our center, the first one at time of first medical consultation and the last at time of last clinical follow-up/enrollment phone call. GFD adherence at time of transition, during follow-up medical consultations at our center, and at enrollment phone call was measured by means of a standard five-level score that was previously developed and validated by our group [33]. Patients scoring from 0 to 2 were considered not adherent, while patients scoring 3-4 were considered adherent. As this score was only developed and validated in 2008, patients seen before 2008 were classified as adherent or not adherent on the basis of a structured dietary interview performed at 
that time by a dietitian or a gastroenterologist with expertise in CD. Patients changing their GFD score during follow-up at our center from adherent to not adherent, or vice versa, were considered to have changed their level of adherence [14].

Clinical and demographic factors, both at baseline and during follow-up, were evaluated for association with longterm GFD adherence. Changes in GFD adherence from the pediatric setting to first adult medical consultation and in the long-term after referral to our center were also evaluated. Predictors of long-term adherence to a GFD were evaluated.

\section{Follow-Up at an Adult Tertiary Referral Center}

For the purpose of this study, follow-up at our adult center was considered regular if patients had attended at least one outpatient medical consultation in the last two years; otherwise, they were considered lost to follow-up. This cutoff was chosen as intermediate between what is commonly accepted for adult CD (annual follow-up consultation) [1-3], the reported literature by other groups for celiac patients diagnosed in the pediatric setting (follow-up during adulthood was considered regular every 3 years) [9], and our clinical experience.

\section{Quality of Life During Adulthood}

QOL was evaluated by means of the Italian version of Celiac Disease Questionnaire (CDQ), a disease-specific healthrelated QOL questionnaire [34]. The overall questionnaire score and the score for each domain were evaluated for association with clinical and demographic factors, both at baseline and during follow-up, and type of transition to adult care.

\section{Statistical Analysis}

$\mathrm{R}$ version 3.6.3 ( $\mathrm{R}$ Core Team 2020, R: A language and environment for statistical computing. R Foundation for Statistical Computing, Vienna, Austria). URL https://www.Rproject.org/) was used for statistical analysis. Categorical variables were summarized via descriptive statistics (total count and percentages) and compared among groups with Fisher's exact test. Median and interquartile range of continuous variables were calculated. Testing for normality of data was performed using the Shapiro-Wilk normality test. Univariate analysis of continuous variables among groups was performed using the Mann-Whitney $U$ test. A $p$ value $<0.05$ was considered statistically significant. Variables for multivariate logistic regression analysis were selected based on clinical relevance among those with $p<0.1$ on univariate analysis. Adjusted odds ratios (OR) and 95\% confidence intervals $(\mathrm{CI})$ were calculated.

\section{Results}

Between January and October 2020, clinical data of 219 adult patients with a pediatric diagnosis of $\mathrm{CD}$ were reevaluated. Thirty-six of them were directly diagnosed at our center during adolescence and were excluded, as they had no transition to adult care. The remaining 183 (130 females, mean age at diagnosis $7.6 \pm 5.8$ years) were considered (Table 1).

\section{Timing of Transition to Adult Care}

Median age at transition to adult care was 20 years (IQR 17-25 years). There was no relationship between age at transition to adult care and long-term GFD adherence $(p=0.63)$, QOL (Spearman rank correlation, Rho $=0.04, p=0.7$ ), and continuity of follow-up $(p=0.07)$. Also, duration of disease did not correlate with these outcomes.

\section{Gluten-Free Diet Adherence}

\section{Pediatric Care}

Results for GFD adherence during pediatric care were available in 164/183 patients: adherence was good in 102 (Table 1).

\section{First Adult Medical Consultation at Our Center}

121/183 patients (66.1\%) had good GFD adherence at first adult consultation (Table 1). Good GFD adherence during pediatric care was strongly related with good adherence at first adult medical consultation (OR 27.90, 95\% CI 11.18-76.59, $p<0.01$ ), whereas clinical pattern of CD and age at diagnosis were not (Table 2). Patients with good adherence at first adult medical consultation were significantly less likely to have persistence of positive EmA (OR $0.19,95 \%$ CI $0.07-0.49, p<0.01)$ and persistence of VA on follow-up duodenal biopsy (OR 0.09, 95\% CI 0.01-0.42, $p<0.01$ ).

\section{Long-Term Adherence to a Gluten-Free Diet and Changes in Adherence During Follow-Up}

Results of long-term GFD adherence were obtained by phone interview in 139/183 patients and at medical consultation in 44/183 patients. Overall, at long-term follow-up, 
Table 1 Factors associated with GFD adherence first medical consultation

\begin{tabular}{|c|c|c|c|c|c|}
\hline & $\begin{array}{l}\text { TOTAL } \\
(n=183)\end{array}$ & $\begin{array}{l}\text { Poor GFD adherence at first } \\
\text { adult medical consultation } \\
N=62\end{array}$ & $\begin{array}{l}\text { Good GFD adherence at first } \\
\text { adult medical consultation } \\
N=121\end{array}$ & $p$ value & OR $(95 \% \mathrm{CI})$ \\
\hline \multicolumn{6}{|l|}{ Factors at baseline } \\
\hline Abdominal pain & $39(23.1 \%)$ & $17(27.4 \%)$ & $22(18.2 \%)$ & 0.18 & \\
\hline Anemia & $33(18.0 \%)$ & $10(16.1 \%)$ & $23(19.0 \%)$ & 0.69 & \\
\hline Autoimmunity & $28(15.3 \%)$ & $9(14.5 \%)$ & $19(15.7 \%)$ & 1.00 & \\
\hline Constipation & $9(4.9 \%)$ & $4(6.5 \%)$ & $5(4.1 \%)$ & 0.49 & \\
\hline Dermatitis herpetiformis & $7(3.8 \%)$ & $3(4.8)$ & $4(3.3)$ & 0.69 & \\
\hline Type of diagnosis & & & & $<0.01$ & - \\
\hline${ }^{\circ}$ Conventional & $169(92.3 \%)$ & $53(85.5 \%)$ & $116(95.9 \%)$ & & \\
\hline${ }^{\circ}$ Only clinical & $8(4.4 \%)$ & $7(11.3 \%)$ & $1(0.8 \%)$ & & \\
\hline${ }^{\circ}$ Serological (ESPHGAN) & $6(3.3 \%)$ & $2(3.2 \%)$ & $4(3.3 \%)$ & & \\
\hline Diarrhea & $82(44.8 \%)$ & $33(53.2 \%)$ & $49(40.5 \%)$ & 0.12 & \\
\hline Dyspepsia & $30(16.4 \%)$ & $10(16.1 \%)$ & $20(16.5 \%)$ & 1.00 & \\
\hline Failure to thrive & $85(46.4 \%)$ & $23(37.1 \%)$ & $62(52.1 \%)$ & 0.09 & \\
\hline Fever & $3(1.6 \%)$ & $1(1.6 \%)$ & $2(1.7 \%)$ & 1.00 & \\
\hline Gender $(F)$ & $130(71.0 \%)$ & $39(62.9 \%)$ & $91(75.2 \%)$ & 0.09 & \\
\hline HLA * & & & & 0.32 & \\
\hline${ }^{\circ} \mathrm{DQ} 2$ & $25(80.6 \%)$ & $11(73.3 \%)$ & $14(87.5 \%)$ & & \\
\hline${ }^{\circ} \mathrm{DQ} 8$ & $4(12.9 \%)$ & $3(20.0 \%)$ & $1(6.2 \%)$ & & \\
\hline${ }^{\circ} \mathrm{DQ} 2 / \mathrm{DQ} 8$ & $1(3.2 \%)$ & $1(6.7 \%)$ & 0 & & \\
\hline${ }^{\circ} \mathrm{DQ7} .5$ & $1(3.2 \%)$ & 0 & $1(6.2 \%)$ & & \\
\hline Classical presentation & $125(68.3 \%)$ & $41(66.1 \%)$ & $84(69.4 \%)$ & 0.74 & \\
\hline Weight loss & $27(14.8 \%)$ & $6(9.87 \%)$ & $21(17.4 \%)$ & 0.19 & \\
\hline Family history of CD & $35(19.1 \%)$ & $10(16.1 \%)$ & $25(20.7 \%)$ & 0.55 & \\
\hline $\begin{array}{l}\text { Age at diagnosis (years, } \\
\text { median, IQR) }\end{array}$ & $8(1.00-13.00)$ & $7.50(1.00-13.00)$ & $9.00(2.00-13.00)$ & 0.15 & \\
\hline \multicolumn{6}{|l|}{ Factors during follow-up } \\
\hline $\begin{array}{l}\text { Age at first adult medical } \\
\text { consultation (years, median, } \\
\text { IQR) }\end{array}$ & $20(17.00-25.00)$ & $21.00(17.00-26.00)$ & $20.00(17.00-23.00)$ & 0.34 & \\
\hline Pediatric GFD adherence * & & & & $<0.01$ & $27.90(11.18-76.59)$ \\
\hline${ }^{\circ}$ Good & $102(62.2 \%)$ & $10(17.5 \%)$ & $92(86.0 \%)$ & & \\
\hline${ }^{\circ}$ Poor & $62(37.8 \%)$ & $47(82.5 \%)$ & $15(14.0 \%)$ & & \\
\hline $\begin{array}{l}\text { Persisting symptoms at first } \\
\text { adult consultation }\end{array}$ & $74(40.4 \%)$ & $28(45.2 \%)$ & $46(38.0 \%)$ & 0.43 & \\
\hline Persistence of positive EmA* & $34(31.5 \%)$ & $24(51.1 \%)$ & $10(16.4 \%)$ & $<0.01$ & $0.19(0.07-0.49)$ \\
\hline Persistence of villous atrophy* & $18(21.2 \%)$ & $16(37.2 \%)$ & $2(4.8 \%)$ & $<0.01$ & $0.09(0.01-0.42)$ \\
\hline $\begin{array}{l}\text { Number of adult medical con- } \\
\text { sultations (median, IQR) }\end{array}$ & $3(1.00-5.00)$ & $3(2.00-5.00)$ & $2(1.00-4.00)$ & 0.08 & \\
\hline $\begin{array}{l}\text { Follow-up duration at our } \\
\text { center (months, median, } \\
\text { IQR) }\end{array}$ & $12.00(0.00-60.50)$ & $14.50(1.25-70.00)$ & $10(0.00-52.00)$ & 0.08 & \\
\hline $\begin{array}{l}\text { Months since last medical con- } \\
\text { sultation (median, IQR) }\end{array}$ & $80(23-138)$ & $106.5(46.75-149.50)$ & $67.00(21.00-129.00)$ & 0.08 & \\
\hline Lost to follow-up ${ }^{\S}$ & $135(73.8 \%)$ & $49(79.0 \%)$ & $86(71.1 \%)$ & 0.29 & \\
\hline $\begin{array}{l}\text { QOL questionnaire score } \\
\text { (median, IQR) ** }\end{array}$ & $167.00(156.50-178.00)$ & $162.00(153.00-173.00)$ & $169.00(159.25-179.75)$ & 0.16 & \\
\hline
\end{tabular}

*Data not available for some patients: no HLA for 152, no pediatric GFD adherence for 19, no duodenal biopsy for 98, no EmA for 75: percentages were calculated excluding missing data points

** There was also no significant difference for any of the four domains of the questionnaire between groups

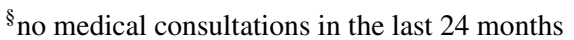


Table 2 Factors associated with long-term GFD adherence during adult follow-up

$\begin{array}{ll}\begin{array}{l}\text { Poor long-term GFD adherence } \\ (n=42)\end{array} & \begin{array}{l}\text { Good long-term } \\ \text { GFD adherence } \\ (n=141)\end{array}\end{array}$

Factors at diagnosis

Abdominal pain

Anemia

$11(26.2 \%)$

$5(11.9 \%)$

Aphthous stomatitis

0

Asthenia

$5(11.9 \%)$

Autoimmunity

$7(16.7 \%)$

Constipation

$2(4.8 \%)$

Dermatitis herpetiformis

0

$\begin{array}{ll}28(19.9 \%) & 0.40 \\ 28(19.9 \%) & 0.36 \\ 12(8.5 \%) & 0.07 \\ 10(7.1 \%) & 0.34 \\ 21(14.9 \%) & 0.81 \\ 7(5.0 \%) & 1.00 \\ 7(5.0 \%) & 0.35 \\ & 0.52\end{array}$

Type of diagnosis

${ }^{\circ}$ Conventional

$40(95.2 \%)$

$129(91.5 \%)$

${ }^{\circ}$ Only clinical

$2(4.9 \%)$

o Serological (ESPHGAN)

0

Diarrhea

$18(42.9 \%)$

$6(4.3 \%)$

$6(4.3 \%)$

Dyspepsia

$7(16.7 \%)$

Failure to thrive

$18(42.9 \%)$

Fever

$1(2.4 \%)$

Gender (F)

$28(66.7 \%)$

$64(45.4 \%)$

$23(16.3 \%)$

0.86

$66(47.5 \%)$

1.00

$2(1.4 \%)$

0.73

HLA*

$6(66.7 \%)$

$102(72.3 \%)$

0.55

${ }^{\circ} \mathrm{DQ} 2$

$2(22.2 \%)$

$1(11.1 \%)$

${ }^{\circ} \mathrm{DQ} 2 / \mathrm{DQ} 8$

0

${ }^{\circ} \mathrm{DQ7} .5$

Classical presentation

$23(54.8 \%)$

$5(11.9 \%)$

$11(26.2 \%)$

Family history of CD

$4(1.0-11.0)$

$19(86.4 \%)$

0

$1(4.5 \%)$

$2(9.1 \%)$

$102(72.3 \%) \quad 0.04$

$2.15(0.99-4.65)$

$22(15.6 \%) \quad 0.63$

$24(17.0 \%) \quad 0.19$

Age at diagnosis (years, median, IQR)

$9(1.0-13.0)$

0.09

Factors during pediatric follow-up, transition and adult follow-up

Good GFD adherence during pediatric follow-up*

$15(41.7 \%)$

$20(17.0-23.75)$

$19(45.2 \%)$

$9(34.6 \%)$

$7(30.4 \%)$

$2.0(1.0-4.75)$

$273.0(218.0-458.0)$

$7.50(0.00-66.25)$

$111.0(47.0-149.5)$

$37(88.1 \%)$

$173.5(158.5-182.0)$
$87(68.0 \%)$

$<0.01 \quad 2.95(1.30-6.85)$

$20(17.0-25.0)$

0.63

$102(72.3 \%)$

$25(30.5 \%)$

$<0.01 \quad 3.14(1.46-6.86)$

$11(17.7 \%)$

0.81

0.24

$3.0(1.0-5.00)$

0.26

$269.0(166.0-413.0) \quad 0.19$

$15.00(0.00-60.00) \quad 0.66$

$70.0(21-0-130.0) \quad 0.06$

$98(69.5 \%) \quad 0.02$

$166.0(156.0-175.5) \quad 0.32$

QOL questionnaire score (median, IQR) **

$C D$ celiac disease, $F$ females, $V A$ villous atrophy, $G F D$ gluten-free diet, $E m A$ endomysial antibodies, $I Q R$ interquartile range; ${ }^{\S}$ no medical consultations in the last 24 months

*Data not available for some patients: no HLA for 152, no pediatric GFD adherence for 19, no duodenal biopsy for 98, no EmA for 75: percentages were calculated excluding missing data points

** There was also no significant difference for any of the four domains of the questionnaire between groups

141/183 (77.0\%) patients had good GFD adherence, while 42/183 (23.0\%) were poorly adherent. 25/164 patients (15.2\%) changed their GFD adherence between pediatric follow-up and first adult medical consultation (Figure 1).
Adherence tended to overall improve slightly from pediatric follow-up to first adult medical consultation, with 15/62 ( $24.1 \%)$ poorly adherent patients improving their degree of 
Figure 1 Patients who changed their level of adherence to a gluten-free diet over time. 25/164 patients changed their GFD adherence between pediatric follow-up and first adult medical consultation $(15 / 62$ poorly adherent patients improved and 10/102 worsened their adherence). GFD adherence changed during adult follow-up in 58/183 patients (39/62 poorly adherent patients at first adult medical consultation became adherent over the long-term, while 19/121 strictly adherent patients became poorly adherent). In the long-term 141/183 patients had good GFD adherence. GFD gluten-free diet, $N A$ not available

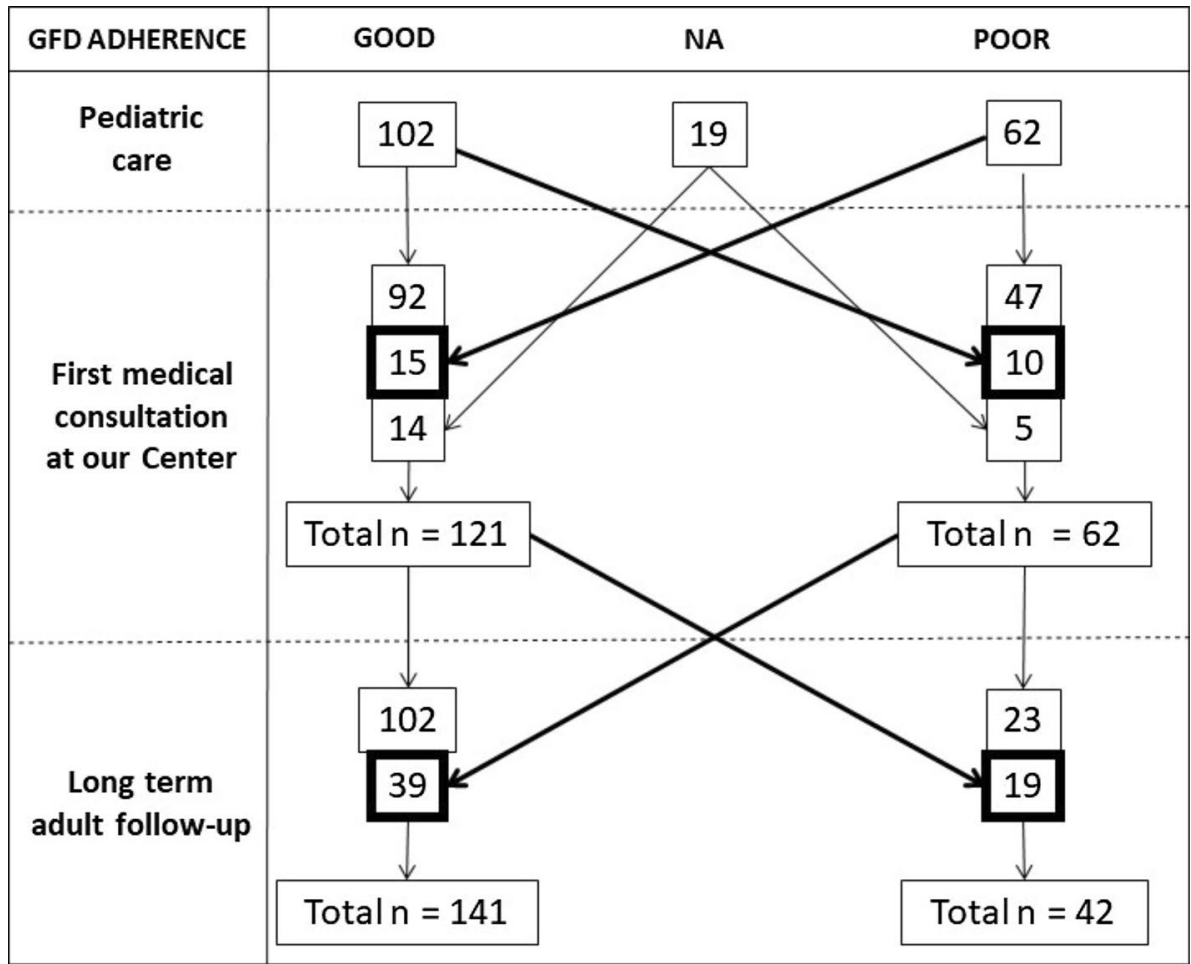

adherence, while only 10/102 (9.8\%) worsened their adherence (OR 2.92, 95\% CI 1.13-7.87, $p=0.02$ ).

GFD adherence changed during adult follow-up at our center in $58 / 183$ patients (31.7\%), with $39 / 62$ (62.9\%) poorly adherent patients at first adult medical consultation becoming adherent over the long-term, while 19/121 (19.0\%) strictly adherent patients at first adult medical consultation became poorly adherent in the long-term. Improvement in adherence during adult follow-up was much more common than worsening (OR 9.0, 95\% CI 4.2-19.7, $p<0.01$ ).

\section{Determinants of Long-Term Gluten-Free Diet Adherence}

On univariate analysis (Table 2), classical presentation of CD, pediatric GFD adherence, and GFD adherence at first adult medical consultation were associated with long-term GFD adherence. Patients with good long-term adherence were less likely to have been lost to follow-up than those with poor adherence, although there was a high proportion of patients lost to follow-up in both groups. Table 4 shows results of multivariable logistic regression analysis: classical presentation of CD (OR 2.37, 95\% CI 1.12-5.04, $p=0.02$ ) and good GFD adherence at first adult medical consultation (OR 3.19, 95\%
CI 1.53-6.66, $p<0.01)$ were predictive of good long-term GFD adherence, while being lost to follow-up was predictive of poorer long-term GFD adherence (OR 0.29, 95\% CI $0.10-0.84, p=0.02$ ).

\section{Maintenance of Regular Follow-Up During Adulthood}

Table 3 shows characteristics of patients in regular followup at our center compared to those lost to follow-up. In our study population, the majority of patients were lost to follow-up. Patients were generally lost to follow-up for a significant time (median 119 months, IQR 67.5-167.5). Figure 2 shows distribution of patients according to time since last medical consultation at our center. Patients lost to follow-up had poorer pediatric GFD adherence and poorer long-term GFD adherence, although GFD adherence at time of transition was not significantly different among those lost to follow-up and those still regularly followed-up. Duration of disease was much longer in patients lost to follow-up than in those still in regular follow-up. 
Table 3 Characteristics of patients in regular follow-up (at least one medical consultation in the last 24 months) and patients lost to follow-up (no medical consultation for $>24$ months)

In regular follow-up $\quad$ Lost to follow-up $(n=135) \quad p$ value OR (95\% CI )
$(n=48)$

\section{Factors at diagnosis}

Classical presentation

Age at diagnosis (years, median, IQR)

Factors at follow-up

Age at first adult medical consultation (years, median, IQR)

Good GFD adherence during pediatric follow-up*

Good GFD adherence at first adult consultation

Good GFD adherence at last contact

Persistence of positive EmA *

Persistence of VA*

Number of adult medical consultations (median, IQR)

Follow-up duration at our center (months, median, IQR)

Months since last medical consultation (median, IQR)

QOL questionnaire score (median, IQR) **

Duration of disease (years, median, IQR)

$\begin{array}{ccrl}33(68.8 \%) & 92(68.1 \%) & 1.00 & \\ 10.0(5.0-13.0) & 7.0(1.0-13.0) & 0.15 & \\ 18.5(16.0-21.5) & 21.0(17.0-25.0) & & \\ & & & \\ 35(76.1 \%) & 67(56.8 \%) & 0.07 & 0.42(0.17-0.94) \\ 35(72.9 \%) & 86(63.7 \%) & 0.29 & \\ 43(89.6 \%) & 98(72.6 \%) & 0.02 & 0.31(0.09-0.87) \\ 4(16.0 \%) & 30(36.1 \%) & 0.08 & \\ 0 & 18(27.3 \%) & <0.01 & \text { NA } \\ 3.0(2.0-5.75) & 2.0(1.0-4.0) & <0.01 & \text { NA } \\ 29.0(0.75-111.25) & 11.0(0.0-43.0) & 0.02 & \\ 10.0(4.75-17.0) & 119.0(67.5-167.5) & \text { NA } & \\ 167.0(154.5-176.0) & 166.5(157.75-179.25) & 0.97 & \\ 13.5(9.00-26.25) & 24.0(18.00-37.00) & <0.01 & \end{array}$

$G F D$ gluten-free diet, $V A$ villous atrophy, $E m A$ endomysial antibodies, $Q O L$ quality of life, $I Q R$ interquartile range

*Data not available for some patients: no pediatric GFD adherence for 19, no duodenal biopsy for 98, no EmA for 75: percentages were calculated excluding missing data points

** There was also no significant difference for any of the four domains of the questionnaire between groups

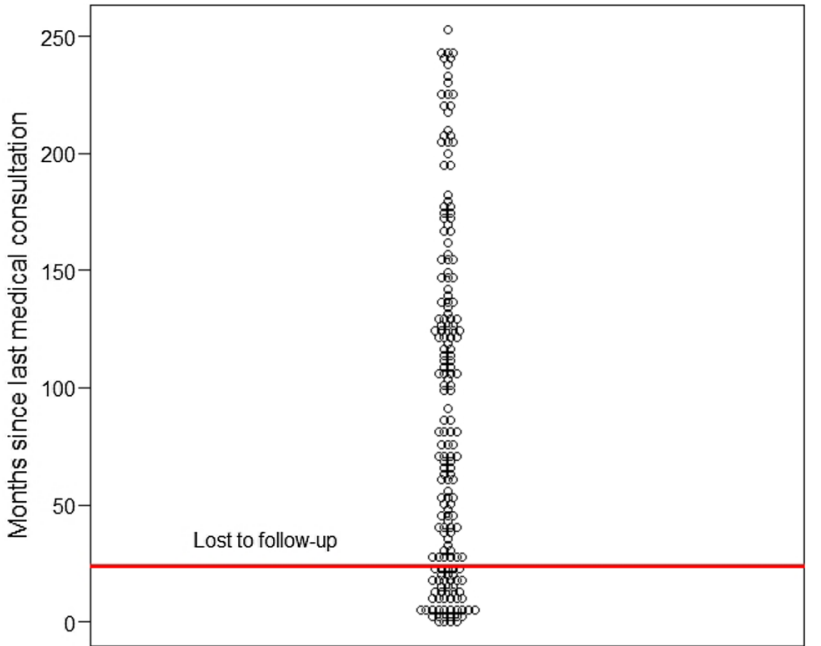

Figure 2 Dot plot showing time since last medical consultation. Horizontal red line is set at 24 months, which was considered the cutoff for being lost to follow-up

\section{Quality of Life}

One-hundred-and-thirty-nine patients $(76.0 \%)$ were contacted over the phone for participation in the QOL study and sent the questionnaire, while $44(24.0 \%)$ patients could not be contacted. Of these 139 patients, 95 (68.3\%) completed and returned the questionnaire. No relationship
Table 4 Multivariate analysis on predictors of successful early transition, long-term adherence to a GFD and regular long-term follow-up

\begin{tabular}{lll}
\hline Factor & Adjusted OR (95\% CI) & $p$ value \\
\hline $\begin{array}{l}\text { Predictors of long-term GFD } \\
\text { adherence }\end{array}$ & & \\
$\begin{array}{l}\text { Good GFD adherence at first adult } \\
\text { medical consultation }\end{array}$ & $3.19(1.53-6.66)$ & $<0.01$ \\
$\begin{array}{l}\text { Classical presentation } \\
\text { Lost to follow-up }\end{array}$ & $2.37(1.12-5.04)$ & 0.02 \\
\hline
\end{tabular}

$C D$ celiac disease, $G F D$ gluten-free diet, $O R$ odds ratio

between QOL and GFD adherence during pediatric care, at time of transition and over the long-term was found (Table 3). No relationship was found with continuity of follow-up, either (Table 4).

\section{Discussion}

This study provides a real-life clinical overview on followup during adult care of patients with CD diagnosed during childhood/adolescence. Patterns of long-term GFD adherence, predictors of long-term GFD adherence and continuity of follow-up were described.

Due to the paucity of data on this subject, organizing a successful transition to adult care in celiac patients is still 
challenging [1-3, 9, 21-30]. We have shown that timing of transition (both age at referral to adult care and duration of disease) is not related to long-term GFD adherence, QOL, and maintenance of regular follow-up during adulthood. It is nevertheless noteworthy that patients' GFD adherence improves after transition to adult care.

We found that good GFD adherence during pediatric care was related to good GFD adherence at first adult medical consultation and over the long-term. Moreover, only a minority of patients changed their level of adherence from childhood to adulthood and throughout adult follow-up. Adherence tended to improve over time, both from pediatric to adult care, and, even more so, throughout adult care. We identified good GFD adherence at time of adult referral and classical presentation of $\mathrm{CD}$ as independent predictors of good long-term GFD adherence, while being lost to follow-up was predictive of poorer long-term GFD adherence. These results are not surprising and are similar to what we previously reported for patients diagnosed in adulthood and followed-up at a referral center [14]. Changes in GFD adherence over time seem to be more common in patients diagnosed in childhood than in adulthood, although it is difficult to compare data between these two populations.

Less than one third of patients diagnosed during childhood/adolescence and then referred to our unit, maintained regular follow-up at our adult referral center. This confirms previous data on lack of regular long-term follow-up in celiac patients diagnosed in childhood [9, 21, 23-27, 35]. Moreover, patients lost to follow-up generally had a much longer duration of disease than patients still in regular follow-up. A possible explanation could be that patients with a longer disease duration have become used to $\mathrm{CD}$ and do not feel the need to maintain regular follow-up as they have already developed strategies to cope with their disease, albeit with a lesser overall degree of GFD adherence [27].

We did not find any relationship between QOL results and timing of transition, long-term GFD adherence or continuity of follow-up, although QOL was generally good overall.

Limitations of our study include the small sample size, the retrospective and single-center setting in a referral center and the lack of standard measures for assessing GFD adherence in pediatric patients. Since we have described only those patients who were referred to our center, this limits the generalizability of our results, albeit they likely represent a minority of all the celiac patients diagnosed in childhood. Furthermore, we did not have any information on those patients who received a diagnosis of $\mathrm{CD}$ during childhood, but were not referred to our center. Providing an estimate of this unseen group of patients is challenging. We cannot exclude that they maintained regular follow-up at another referral center. However, if we consider the literature $[9,21,23-27,35]$ and the high proportion of patients lacking regular follow-up even at our center, we cannot exclude that many of them were indeed lost to follow-up.
Although it is unlikely for patients diagnosed in childhood to have developed poor long-term outcomes, we could not evaluate development of complications and mortality [5, 8].

Another limit is the lack of objective measures of adherence during pediatric follow-up. In our study, data on pediatric adherence were retrospectively collected, either from what was reported on patients' clinical notes, or by interviewing the patients' and their parents/carers at first medical consultation at our center. However, since methods for assessing GFD adherence in children/adolescents are not standardized, and a meta-analysis has recently shown that these methods perform similarly [36], we believe our results are acceptable.

Finally, diagnoses of CD in our study cohort were made according to different diagnostic criteria due to the timespan of the study. However, this was inevitable and reflects real-life clinical practice in the last two decades.

In conclusion, long-term GFD adherence improves after transition to adult care although this does not appear to be related to timing of transition. QOL is good among patients diagnosed in childhood/adolescence after referral to adult care. We have shown that good GFD adherence at time of adult referral and classical pattern at time of diagnosis CD are predictive of good long-term GFD adherence, while being lost to follow-up was predictive of poorer long-term GFD adherence. Although further studies are needed to confirm our findings, this could be useful to identify patients who may benefit at time of transition from additional interventions to improve their long-term GFD adherence. These strategies could include dietary counseling, stricter follow-up, and underlining the importance of maintaining regular follow-up.

Author's contribution AS and FB planned the study. AS, SM, GR, CdQMAdS, SC collected the data. SM performed the statistical analysis. AS and SM interpreted the data and drafted the manuscript. FB revised the manuscript for important intellectual contents. All the Authors revised and approved the final version of the manuscript.

Funding Open access funding provided by Università degli Studi di Pavia within the CRUI-CARE Agreement. This study received no funding.

\section{Declarations}

Conflict of interest None to declare.

Ethical approval The study protocol was approved by the ethical review board of IRCCS Pavia, ICS Maugeri, (protocol 2381CE, 14/01/2020; extended 03/11/2020). The study protocol conforms to the ethical guidelines of the 1975 Declaration of Helsinki (6th revision, 2008) as reflected in a prior approval by the institution's human research committee. All the patients answering the questionnaire gave their written consent to participate to the study.

Open Access This article is licensed under a Creative Commons Attribution-NonCommercial 4.0 International License, which permits any 
non-commercial use, sharing, adaptation, distribution and reproduction in any medium or format, as long as you give appropriate credit to the original author(s) and the source, provide a link to the Creative Commons licence, and indicate if changes were made. The images or other third party material in this article are included in the article's Creative Commons licence, unless indicated otherwise in a credit line to the material. If material is not included in the article's Creative Commons licence and your intended use is not permitted by statutory regulation or exceeds the permitted use, you will need to obtain permission directly from the copyright holder. To view a copy of this licence, visit http://creativecommons.org/licenses/by-nc/4.0/.

\section{References}

1. Ludvigsson JF, Bai JC, Biagi F et al. Diagnosis and management of adult celiac disease: guidelines from the British Society of Gastroenterology. Gut 2014;63:1210-1228.

2. Al-Toma A, Volta U, Auricchio R et al. European Society for the Study of Celiac Disease (ESsCD) guideline for celiac disease and other gluten-related disorders. United European Gastroenterol J 2019;7:583-613.

3. Rubio-Tapia A, Hill ID, Kelly CP et al. ACG clinical guidelines: diagnosis and management of celiac disease. Am J Gastroenterol 2013;108:656-676.

4. Lebwohl B, Green PHR, Söderling J et al. Association between celiac disease and mortality risk in a Swedish Population. JAMA 2020;323:1277-1285.

5. Biagi F, Corazza GR. Mortality in celiac disease. Nat Rev Gastroenterol Hepatol 2010;7:158-162.

6. Sanders DS, Hurlstone DP, Stokes RO et al. Changing face of adult celiac disease: Experience of a single university hospital in South Yorkshire. Postgrad Med J 2002;78:31-33.

7. Volta U, Caio G, Stanghellini V et al. The changing clinical profile of celiac disease: A 15-year experience (1998-2012) in an Italian referral centre. BMC Gastroenterol 2014;14:194.

8. Biagi F, Schiepatti A, Maiorano G et al. Risk of complications in celiac patients depends on age at diagnosis and type of clinical presentation. Dig Liver Dis 2018;50:549-552.

9. Koskimaa S, Kivelä L, Arvola T et al. Clinical characteristics and long-term health in celiac disease patients diagnosed in early childhood: Large cohort study. Dig Liver Dis 2020;52:1315-1322.

10. Kemppainen T, Kröger H, Janatuinen E et al. Bone recovery after a gluten-free diet:a 5-year follow-up study. Bone 1999;25:355-360.

11. Schiepatti A, Sprio E, Sanders DS et al. Celiac disease and obstetric and gynaecological disorders: Where are we now? Eur J Gastroenterol Hepatol 2019;31:425-433.

12. Zingone F, Swift GL, Card TR et al. Psychological morbidity of celiac disease: A review of the literature. United European Gastroenterol J 2015;3:136-145.

13. Holmes GK, Prior P, Lane MR et al. Malignancy in celiac disease-Effect of a gluten free diet. Gut 1989;30:333-338.

14. Schiepatti A, Maimaris S, Nicolardi ML et al. Determinants and trends of adherence to a gluten-free diet in adult celiac patients on a long-term follow-up (2000-2020). Clin Gastroenterol Hepatol. 2020. https://doi.org/10.1016/j.cgh.2020.12.015.

15. Hall NJ, Rubin G, Charnock A. Systematic review: Adherence to a gluten-free diet in adult patients with celiac disease. Aliment Pharmacol Ther 2009;30:315-330.

16. Abu-Janb N, Jaana M. Facilitators and barriers to adherence to gluten-free diet among adults with celiac disease: A systematic review. J Hum Nutr Diet 2020;33:786-810.

17. Halmos EP, Deng M, Knowles SR et al. Food knowledge and psychological state predict adherence to a gluten-free diet in a survey of 5310 Australians and New Zealanders with celiac disease. Aliment Pharmacol Ther 2018;48:78-86.

18. Kurppa K, Lauronen O, Collin P et al. Factors associated with dietary adherence in celiac disease: A nationwide study. Digestion 2012;86:309-314.

19. Leffler DA, Edwards-George J, Dennis M et al. Factors that influence adherence to a gluten-free diet in adults with celiac disease. Dig Dis Sci 2008;53:1573-1581.

20. Villafuerte-Galvez J, Vanga RR, Dennis M et al. Factors governing long-term adherence to a gluten-free diet in adult patients with celiac disease. Aliment Pharmacol Ther 2015;42:753-760.

21. O'Leary $\mathrm{C}$, Wieneke $\mathrm{P}$, Healy $\mathrm{M}$ et al. Celiac disease and the transition from childhood to adulthood: A 28-year follow-up. Am J Gastroenterol 2004;99:2437-2441.

22. Zingone F, Massa S, Malamisura B et al. Celiac disease: Factors affecting the transition and a practical tool for the transition to adult healthcare. United European Gastroenterol J 2018;6:1356-1362.

23. Reilly NR, Hammer ML, Ludvigsson JF et al. Frequency and predictors of successful transition of care for young adults with childhood celiac disease. J Pediatr Gastroenterol Nutr 2020;70:190-194.

24. Peixoto A, Reis E, Melo A, Trindade E et al. Protocol for the transition of pediatrics for adult medicine in celiac disease: A proposal approach. Eur J Gastroenterol Hepatol 2019;31:729-730.

25. Kivelä L, Hekkala S, Huhtala $\mathrm{H}$ et al. Lack of long-term followup after pediatric-adult transition in celiac disease is not associated with complications, ongoing symptoms or dietary adherence. United European Gastroenterol J 2020;8:157-166.

26. van Koppen EJ, Schweizer JJ, Csizmadia CG et al. Long-term health and quality-of-life consequences of mass screening for childhood celiac disease: A-10-year follow-up study. Pediatrics 2009;123:e582-e588.

27. Norsa L, Branchi F, Bravo $\mathrm{M}$ et al. Celiac disease 30 years after diagnosis: Struggling with gluten-free adherence or gaining gluten tolerance? J Pediatr Gastroenterol Nutr 2018;67:361-366.

28. Ludvigsson JF, Agreus L, Ciacci C et al. Transition from childhood to adulthood in celiac disease: The Prague consensus report. Gut 2016;65:1242-1251.

29. Elli L, Maieron R, Martelossi S et al. Transition of gastroenterological patients from pediatric to adult care: A position statement by the Italian Societies of Gastroenterology. Dig Liver Dis 2015;47:734-740.

30. Brooks AJ, Smith PJ, Cohen R et al. UK guideline on transition of adolescent and young persons with chronic digestive diseases from pediatric to adult care. Gut 2017;66:988-1000.

31. Husby S, Koletzko S, Korponay-Szabó IR et al. European Society for Pediatric Gastroenterology, Hepatology, and Nutrition guidelines for the diagnosis of celiac disease. J Pediatr Gastroenterol Nutr 2012;54:136-160.

32. Meeuwisse GW. Round table discussion. Diagnostic criteria in celiac disease. Acta Paediatr 1970;59:461e3.

33. Biagi F, Bianchi PI, Marchese A et al. A score that verifies adherence to a gluten-free diet: A cross-sectional, multicentre validation in real clinical life. Br J Nutr 2012;108:1884-1888.

34. Marchese A, Klersy C, Biagi F et al. Quality of life in celiac patients: Italian validation of a celiac questionnaire. Eur J Intern Med 2013;24:87-91.

35. Barnea L, Mozer-Glassberg Y, Hojsak I et al. Pediatric celiac disease patients who are lost to follow-up have a poorly controlled disease. Digestion 2014;90:248-253.

36. Myléus A, Reilly NR, Green PHR. Rate, risk factors, and outcomes of nonadherence in pediatric patients with celiac disease: A systematic review. Clin Gastroenterol Hepatol 2020;18:562-573.

Publisher's Note Springer Nature remains neutral with regard to jurisdictional claims in published maps and institutional affiliations. 